\section{P84 APNOEIC EVENT IN WOLF-HIRSCHHORN SYNDROME: COULD THIS HAVE BEEN A 'TET' SPELL?}

Kene Maduemem*, Gheetha Arumugam, Amol Chingale. Lincoln County Hospital, Lincoln UK

10.1136/archdischild-2019-epa.439

Background Wolf-Hirschhorn syndrome (WHS) is a rare genetic condition caused by partial deletion of short arm of chromosome 4. Clinical manifestations comprise but not limited to typical facie, mental retardation, growth delay, epilepsy, and congenital cardiac defects. Congenital heart defects occur in about $25-50 \%$ of cases. We describe a child with WHS presenting with a femoral fracture; he became apnoeic in the course of management resulting in a cascade of events.

Case Report 4.5-year-old boy known to have WHS was admitted with left femoral fracture. The syndrome was an antenatal diagnosis. Other associations noted were severe growth delay (weight of $6 \mathrm{~kg}$ ), epilepsy, global developmental delay, visual impairment, and tetralogy of Fallot (TOF). His TOF has been managed uneventfully on propranolol with acceptable oxygen saturation $(\mathrm{Sp02})$ in the 70s. The spontaneous fracture required Gallows traction, which provoked distress. He developed hypoxic crisis requiring oxygen therapy to maintain acceptable SpO2. Subsequently, he went apnoeic and dusky needing bag and mask ventilation. Blood gas revealed severe metabolic acidosis, which improved after fluid bolus. Morphine dosing regimen for pain control was continued. Kneechest position could not be attempted due to the fracture. Although he got treated for suspected sepsis due to the dilemma of events, he did have a hypercyanotic spell in hindsight. He was subsequently discharged with conservative management of the fracture.

Conclusion To the best of our knowledge, this is the first reported case of hypercyanotic (Tet) spell in WHS. This rather uncommon event resulted in a significant learning point for medical and nursing staff.

\section{P85 21 MONTH OLD INFANT WITH SEVERE HYPONATREMIA CAUSED BY EXCESSIVE WATER INTAKE DURING FEBRILE INFECTION. MANAGEMENT AND DISCUSSION OF THE CASE}

${ }^{1}$ Anastasia Anastasiou-Katsiardani* ${ }^{1}$ Konstantinos Tsakos, ${ }^{1}$ Angeliki Beslika, ${ }^{1}$ Maria Lampri, 'Kalliopi Tanou, ${ }^{2}$ Michail Kastamoulas, ${ }^{2}$ Evaggelia Lykopoulou. ${ }^{1}$ Paediatric Ward, 'Achillopouleio' General Hospital of Volos, County of Magnesia, Volos, Greece, ' ${ }^{2}$ irst Department of Paediatrics, 'Agia Sofia Childrens' Hospital', Athens, Greece

10.1136/archdischild-2019-epa.440

Introduction Hyponatremia is the most common electrolyte disorder, defined as a serum sodium level less than $135 \mathrm{meq} /$ L. It is classified according to serum sodium concentration as mild, moderate and severe and according to its manifestation time in acute ( $<48$ hours) and chronic. It can lead to a wide range of symptoms, from mild to severe, even life-threatening (cerebral edema).

Purpose Discussion and management of a case of a 21 month old female infant with severe hyponatremia (Na: $117 \mathrm{meq} / \mathrm{L}$ ), presented with drowsiness accompanied by polydipsia and polyuria following a febrile infection.

Clinical case The infant presented with drowsiness started 12 hours ago, accompanied by polydipsia (water intake $>7 \mathrm{Lt} /$ day), polyuria and high temperature up to $39.8^{\circ} \mathrm{C}$ that started
3 days ago. From the medical history high water intake is reported from the age of 6 months old. At the time of admission, she was haemodynamically stable, afebrile, presented with tonsillitis and signs of mild dehydration. Laboratory tests revealed severe hyponatremia (Na: $117 \mathrm{meq} / \mathrm{L}$ ), dehydration with normal renal function tests, low serum and urine osmolarity and normal white blood cell count and CRP. Kidneyureter-urinary bladder-adrenal gland ultrasound and fundoscopy showed no pathological findings. The patient was treated with intravenous fluids based on the hypotonic dehydration treatment protocol, with continuous neurological, blood gas and biochemical monitoring. Serum sodium levels recovered to normal (Na: $136 \mathrm{meq} / \mathrm{L}$ ) over the following 24 hours. On the third day of hospitalization she was transferred to the Childrens' Hospital of Athens 'Agia Sofia' in order to complete further investigations to exclude possible adrenal or pituitary gland disfunction and cystic fibrosis disease, which revealed no pathological findings. As a result, the most likely cause of hyponatremia was considered to be the excessive water intake.

Conclusions We presume high water intake as the most likely cause of hyponatremia for our patient, after having excluded other causes of hyponatremia. Although high fluid intake is considered to be a rare cause of hyponatremia in childhood, based on published data, it has to be included in the differential diagnosis. The detailed medical history, the clinical examination and laboratory tests lead to early diagnosis. The cautious correction of serum sodium levels, as well as of co-existent acid-based disorders, is the mainstay of successful treatment of such a potentially life-threatening condition

\section{P86 IDIOPATHIC INTERSTITIAL NEPHRITIS (IN) LEADING TO END STAGE KIDNEY DISEASE (ESKD) IN A 17-YEAR-OLD MALE}

James Dillon*, Eoin Bergin, Shodan Osman, Syed Ali Aown. Midlands Regional Hospital Tullamore, Tullamore, Ireland

\subsection{6/archdischild-2019-epa.441}

Background IN, a clinical syndrome of numerous aetiologies including infectious, medication and sometimes idiopathic, is a common cause of acute kidney injury, which is often under-diagnosed. Its incidence has decreased over the last number of years which is thought to be due to the increase use and early implementation of antimicrobials in the, given that infection is one of its leading causes, (Raina, et al., 2016). IN is associated with an immune-mediated infiltration which can lead to fibrosis of the interstitium and ultimately cause chronic kidney disease. Early diagnosis is key to limit renal impairment. Presentation can be quite non-specific: patients often complaining of fatigue or malaise over a number of weeks. This non-specific nature delays diagnosis and ultimately a progression in renal impairment. Treatment is highly dependent on the underlying aetiology, but IN has shown to be quite responsive to steroids. The prognosis of IN depends on the underlying cause as well as timing of intervention. Evidence of a chronic course as well as gross proteinuria, indicate of a poorer prognosis. Biopsy proven renal fibrosis represents irreversible change, (Joyce, et al., 2018). Unfortunately, a delayed diagnosis has the potential to cause ESKD, (Nangaku, 2004). 